\title{
Statics and dynamics of the ten-state nearest-neighbor Potts glass on the simple-cubic lattice
}

Claudio Brangian ${ }^{1}$, Walter $\mathrm{Kob}^{2}$, and Kurt Binder ${ }^{1}$

${ }^{1}$ Institut für Physik, Johannes-Gutenberg-Universität Mainz, Staudinger Weg 7, D-55099 Mainz, Germany

${ }^{2}$ Laboratoire des Verres, Université Montpellier II, F-34095 Montpellier, France

We present the results of Monte Carlo simulations of two different Potts glass models with short range random interactions. In the first model a $\pm J$-distribution of the bonds is chosen, in the second model a Gaussian distribution. In both cases the first two moments of the distribution are chosen to be $J_{0}=-1, \Delta J=+1$, so that no ferromagnetic ordering of the Potts spins can occur. We find that for all temperatures investigated the spin glass susceptibility remains finite, the spin glass order parameter remains zero, and that the specific heat has only a smooth Schottky-like peak. These results can be understood quantitatively by considering small but independent clusters of spins. Hence we have evidence that there is no static phase transition at any nonzero temperature. Consistent with these findings, only very minor size effects are observed, which implies that all correlation lengths of the models remain very short. We also compute for both models the time auto-correlation function $C(t)$ of the Potts spins. While in the Gaussian model $C(t)$ shows a smooth uniform decay, the correlator for the $\pm J$ model has several distinct steps. These steps correspond to the breaking of bonds in small clusters of ferromagnetically coupled spins (dimers, trimers, etc.). The relaxation times follow simple Arrhenius laws, with activation energies that are readily interpreted within the cluster picture, giving evidence that the system does not have a dynamic transition at a finite temperature. Hence we find that for the present models all the transitions known for the mean-field version of the model are completely wiped out. Finally we also determine the time auto-correlation functions of individual spins, and show that the system is dynamically very heterogeneous.

PACS numbers: 64.70.Pf, 75.10.Nr, 75.50.Lk, 02.70.Lq 


\section{INTRODUCTION}

Recently much attention has been given to the study of several models of mean field spin glasses [1, 2, 3, 4, such as the spin glasses with $p$-spin interaction [5, 6]

and $p$-state Potts spin glasses with $p>4$ [1, 7, 8, 9, 10, 11, 12, 13, 14, 15, 16, 17, 18, 19]. The interest in these latter models is related to the fact that they exhibit two distinct transitions: A dynamic transition from ergodic to non-ergodic behavior at a temperature $T_{D}$ where the phase space of the systems splits into many valleys separated by infinitely high barriers of the free energy, and a static transition at a temperature $T_{0}<T_{D}$. Although at the static transition a (static) glass order parameter appears discontinuously and the internal energy and entropy have kink singularities, there is no latent heat (Fig. 1). Due to these properties it has been proposed that these spin glass models are generic models for the structural glass transition from a supercooled fluid to an amorphous solid [20, 21, 22, 23, 24, 25]. In fact, the slowing down of the relaxation near $T_{D}$ is described by the same type of equations [9, 10, 11, 15 as has been proposed before in the framework of the so-called idealized mode coupling theory (MCT) [26, 27, 28, 29] to describe the onset of the slowing down in supercooled fluids near the glass transition, and it is known that MCT describes a variety of experiments and simulations quite successfully [27, 29, 30]. In addition, the $T$-dependence of the entropy of these models (Fig. 1) is reminiscent of the behavior of the entropy of supercooled fluids near the glass transition, which can be extrapolated to the Kauzmann temperature $T_{K}$ (which is below the experimental glass transition temperature $T_{g}$ ) [31] at which it seems to be equal to the entropy of the corresponding crystal. Since one does not expect that the entropy of the (disordered) fluid is less than the entropy of the (ordered) crystal, one often invokes a static phase transition from the (metastable) supercooled fluid to a (metastable) ideal glass at a temperature $T_{0} \geq T_{K}$ [32, 33, 34]. However, the existing theories for such a static transition underlying the structural glass transition rely on mean-field type approximations whose validity is questionable [35, 36]. Since real systems have short range interactions, it is relevant to ask which features of the mean field Potts glass (the scenario of Fig. 1 holds true for infinite range interactions) can also be found in systems with short range interactions. In fact, one must expect that in a short range system the temperature $T_{D}$ can only have the character of a crossover temperature, where valleys in phase space appear that are separated by barriers of large but finite height. Such a scenario is also suggested by the extended form of MCT 28 and is certainly also required from an analysis of experimental data [29] which clearly rules out a true power-law divergence of the relaxation time at $T_{D}$ in supercooled fluids. Instead one finds in experiments a smooth crossover from an apparent power-law for $T>T_{D}$ to an activated behavior (Arrhenius-behavior: $\ln \tau \propto 1 / T$ ) for $T<T_{D}$ or an even stronger $T$-dependence. Of course, if the singularities at $T_{D}$ are rounded off in systems with short range interactions it is of interest to ask whether the static transition at $T_{0}$ is still observed or is also wiped out by the fluctuations that are neglected in mean field theories. Eastwood and Wolynes 
have recently put forward some arguments by which supposedly it can be decided whether or not a short range model shows close to $T_{0}$ the behavior expected from the mean field theories [37]. However, these arguments rely on the idea that it is possible to partition a given configuration into droplets which have a well-defined free energy and surface tension. To what extend such ideas are indeed applicable also to simple spin glasses with nearest neighbor interactions is presently an open question, however.

The motivation for the present study is to address some of the issues discussed in the previous paragraph by means of numerical simulations. The systems considered are $d=3$ dimensional 10-state Potts glasses with nearest neighbor interactions 38. In Sec. II we will describe the models that are studied in the present paper and define the quantities that are calculated. The main effort of our work is concentrated on the $\pm J$ model, and the results on the static and dynamic properties of that model will be presented in Secs. III and [V]. Finally, in Sec. D we will present some results on the Gaussian model, while in Sec. VI we summarize our conclusions.

\section{MODELS AND DETAILS ON THE SIMULATION}

In this section we define the systems that are studied in the present work and introduce the observables that we will consider. Also some details on our simulation procedures are given.

The Hamiltonian of the $p$-state Potts glass is given by

$$
\mathcal{H}=-\sum_{\langle i, j\rangle} J_{i j}\left(p \delta_{\sigma_{i} \sigma_{j}}-1\right)
$$

where the "exchange constants" $J_{i j}$ are quenched random variables, and the symbol $\langle i, j\rangle$ means that each pair of nearest neighbor sites $i, j$ on the simple cubic lattice is included in the sum only once. In the present work we will always use $p=10$, and will set the Botltzmann constant $k_{B}=1$. Most of our work concentrates on a bimodal distribution

$$
P\left(J_{i j}\right)=x \delta\left(J_{i j}-J\right)+(1-x) \delta\left(J_{i j}+J\right),
$$

since in a simulation of this model the transition probabilities used in the MonteCarlo algorithm for the assignment of a new value of a Potts spin (we used a single spin-flip type algorithm) can be pre-computed and stored in a small table. Hence they need not be recomputed in the course of the simulation, which in turn leads to a faster algorithm.

If we denote the first two moments of the distribution $P\left(J_{i j}\right)$ as $J_{0}$ and $(\Delta J)^{2}$, respectively,

$$
J_{0}=\left[J_{i j}\right]_{\mathrm{av}}, \quad(\Delta J)^{2}=\left[J_{i j}^{2}\right]_{\mathrm{av}}-\left(\left[J_{i j}\right]_{\mathrm{av}}\right)^{2},
$$


we obtain the relation with $x$ and $J$ from Eq. (2) as

$$
x=\frac{1}{2}\left(1+J_{0} / J\right) \quad, \quad J=\sqrt{J_{0}^{2}+(\Delta J)^{2}} .
$$

(Note that the brackets [.] $]_{\mathrm{av}}$ in Eq. (3) stand for the average over the distribution $P\left(J_{i j}\right)$ from Eq. (2). In practice this was done by using typically 100 independent realizations of bond configurations.)

To fix the temperature scale we set $\Delta J=1$ and furthermore we choose $J_{0}=-1$ to avoid the tendency to ferromagnetic ordering. Note that in the Ising spin glass [四] the choice $J_{0}=0$ is most natural and does not lead to any ferromagnetic correlations in that model. However, this is no longer true in Potts glasses with $p>3$ as we will show now: Consider the ferromagnetic susceptibility

$$
\chi_{\mathrm{FM}}=N\left[\left\langle m^{2}\right\rangle\right]_{\mathrm{av}}
$$

where $m$ is the "magnetization" per spin of the Potts model (using the simplex representation discussed below) and $N=L^{3}$ is the number of sites in the $L \times L \times L$ simple cubic lattice. (Note that in order to minimize finite size effects we always used periodic boundary conditions.) The brackets $\langle$.$\rangle stand for the usual canonical$ average which were calculated by standard Monte Carlo averaging [39, 40], using runs of a typical length of $10^{8}$ Monte Carlo steps (MCS) per site. (Before these production runs were started, we equilibrated the samples for the equal amount of time.)

The temperature dependence of $\chi_{\mathrm{FM}}$ for the case $J_{0}=0$ and $J_{0}=-1$ is shown in Figs. 2a and $2 \mathrm{~b}$, respectively. For $J_{0}=0, \chi_{\mathrm{FM}}$ gets rather large at low temperatures and increases rapidly with increasing $L$. In contrast to this the curves for $J_{0}=-1$ stay on the order of unity in the whole temperature range and show, within the statistical errors, no systematic dependence on the lattice size. Hence we conclude that the choice $J_{0}=-1$ does not lead to any ferromagnetic ordering of the system.

According to Eq. (雨) the choice $\Delta J=1$ and $J_{0}=-1$ leads to

$$
x=(1-1 / \sqrt{2}) / 2 \approx 0.146 \text { and } J=\sqrt{2},
$$

and these are the values that we use in this work.

In addition to the $\pm J$ model defined by Eqs. (1) and (2), we also investigate the Gaussian model defined by

$$
P\left(J_{i j}\right)=\frac{1}{\sqrt{2 \pi}(\Delta J)} \exp \left[-\frac{\left(J_{i j}-J_{0}\right)^{2}}{2(\Delta J)^{2}}\right] .
$$

Results for this model will be discussed in Sec. V.

The static quantities determined in our simulations include the energy per spin and the specific heat calculated from energy fluctuations,

$$
e(T)=\frac{1}{N}[\langle\mathcal{H}\rangle]_{\mathrm{av}} \quad \text { and } \quad c(T)=\frac{1}{N T^{2}}\left\{\left[\left\langle\mathcal{H}^{2}\right\rangle\right]_{\mathrm{av}}-\left[\langle\mathcal{H}\rangle^{2}\right]_{\mathrm{av}}\right\}
$$


For defining observables like the magnetization, the glass order parameter, and the time-dependent spin auto-correlation function, etc., it is useful to choose a representation for the spins that takes into account the symmetry between their $p$ possible states. This can be achieved by the so-called "simplex representation" [41, 42], in which each of the $p$ states corresponds to a $(p-1)$-dimensional vector $\vec{S}_{\lambda}$ pointing towards the corner of a $p$-simplex, i.e.

$$
\vec{S}_{\lambda} \cdot \vec{S}_{\lambda^{\prime}}=\left(p \delta_{\lambda \lambda^{\prime}}-1\right) \quad \text { with } \lambda, \lambda^{\prime} \in\{1,2, \cdots, p\} .
$$

To define a spin glass order parameter, we follow the standard method used for Potts glasses [16, 17, 18, 19, 43] by considering two real replicas $\alpha$ and $\beta$ of the model system, i.e. two systems that have identical bond configurations, and to carry out for each of them an independent Monte Carlo simulation. The order parameter tensor is then defined as

$$
q^{\mu \nu}=\frac{1}{N} \sum_{i=1}^{N}\left(\vec{S}_{i, \alpha}\right)^{\mu}\left(\vec{S}_{i, \beta}\right)^{\nu} \quad \mu, \nu \in\{1,2, \cdots, p-1\} .
$$

In an equilibrium simulation of a finite system with no external fields that couple to components of the order parameter, there is no symmetry breaking, and hence it is sufficient to consider the root mean square order parameter [16, 17, 18, 19, 43]:

$$
q=\left[\frac{1}{p-1} \sum_{\mu, \nu=1}^{p-1}\left(q^{\mu \nu}\right)^{2}\right]^{1 / 2}
$$

By calculating a histogram of $q$ (i.e. one always takes first the thermal average and then the sample average over the disorder) we can estimate the distribution $P(q)$ of the order parameter. The various moments of this distribution are then given by

$$
\left[\left\langle q^{k}\right\rangle\right]_{\mathrm{av}}=\int_{0}^{1} q^{k} P(q) d q
$$

The spin glass susceptibility can now be defined in terms of the second moment:

$$
\chi_{\mathrm{SG}}=\frac{N}{p-1}\left[\left\langle q^{2}\right\rangle\right]_{\mathrm{av}}
$$

Further quantities that exhibit interesting behavior if a phase transition occurs are the reduced fourth-order cumulant [16, 17, 18, 19, 43]

$$
g_{4}(N, T)=\frac{(p-1)^{2}}{2}\left(1+\frac{2}{(p-1)^{2}}-\frac{\left[\left\langle q^{4}\right\rangle\right]_{\mathrm{av}}}{\left[\left\langle q^{2}\right\rangle\right]_{\mathrm{av}}^{2}}\right)
$$


and the so-called Guerra parameter 44, 45

$$
G(N, T)=\frac{\left[\left\langle q^{2}\right\rangle^{2}\right]_{\mathrm{av}}-\left[\left\langle q^{2}\right\rangle\right]_{\mathrm{av}}^{2}}{\left[\left\langle q^{4}\right\rangle\right]_{\mathrm{av}}-\left[\left\langle q^{2}\right\rangle\right]_{\mathrm{av}}^{2}} .
$$

Both quantities are defined such that for $N \rightarrow \infty$ they are zero throughout the disordered phase, while they differ from zero in the ordered phase. If the system has a phase transition at a nonzero temperature $T_{c}$, one expects that the curves $g_{4}(N, T)$, or $G(N, T)$, plotted vs. $T$ for different choices of $N$ intersect at a common intersection point at $T=T_{c}$. In particular, $G(N, T)$ is a measure for the lack of self-averaging [44].

To study the dynamical properties of the system, we calculate the time autocorrelation function of the Potts spins,

$$
C(t)=\frac{1}{N(p-1)} \sum_{i=1}^{N}\left[\left\langle\vec{S}_{i}\left(t^{\prime}\right) \cdot \vec{S}_{i}\left(t^{\prime}+t\right)\right\rangle\right]_{\mathrm{av}} \quad .
$$

(Note that in equilibrium, the right hand side of this equation depends only on the time difference $t$.)

It is also of interest to study a time correlation function that is analogous to $C(t)$, in which, however, one considers only the time auto-correlation function of the spin for each lattice site $i$, since such a quantity will yield information on the dynamical heterogeneity [46, 47, 48] of our model system. Below we will investigate these functions $C_{i}(t)$ in detail.

To propagate the system in configuration space, we used the heat bath algorithm [39]. I. e., to every possible state $\ell$ of a Potts spin $(\ell \in\{1,2, \cdots, p\})$ a probability $p_{\ell}$ is assigned,

$$
p_{\ell}=\frac{\exp \left(-E_{\ell} / T\right)}{\sum_{m=1}^{p} \exp \left(-E_{m} / T\right)}
$$

where $E_{\ell}$ is the total energy of the system if the considered Potts spin is in state $\ell$. At each time step a random spin $i$ is picked, the probabilities given by Eq. (17) are determined, and the spin is flipped to state $\ell$ with probability $p_{\ell}$. (Hence the algorithm is quite efficient in finding energetically desirable states even for large values of $p$, in contrast to the standard Metropolis algorithm.) Note that in the present case the computation of $E_{\ell}$ involves only a consideration of the $z=6$ nearest neighbors, unlike the mean field model studied previously 16, 17, 18, 19.

\section{STATIC PROPERTIES OF THE $\pm J$ POTTS GLASS}

In Figs. 3 and 1 we show the temperature dependence of the energy per spin $e(T)$ and the specific heat per spin $c(T)$, Eq. (8). We see that for $T \lesssim 2$ the energy seems to become independent of $T$, i.e. it must be close to the ground state energy 
$e(0) \approx-8.8$. This value for $e(0)$ is not far from the value that one would predict if only the energetically favored ferromagnetic bonds were fully satisfied. In fact, in such a case we would have (note the normalization of $\mathcal{H}$ in Eq. (10))

$$
e(0)=-3[J x(p-1)-J(1-x)(-1)]=-3 J[x p-2 x+1] \approx-9.2 .
$$

This estimate is only $5 \%$ lower than the actually observed value, showing that the effect of frustration in the present model is very small, unlike the case of the $\pm J$ Ising spin glass [1]. Since this frustation is so low, it should be possible to describe the thermodynamic behavior of the system as a superposition of small independent clusters of spins. In the Appendix we present such an approach and give the $T$-dependence of the energy and the specific heat (Eqs. (A.3), (A.4), and (A.6)). The result of this calculation is included in Figs. 3 and 14 as well. We see that for the energy the theoretical curve at low $T$ is somewhat lower than the data from the simulations (since not all effects of the frustration are taken into account) but that for intermediate and high temperatures the agreement is very good.

Figure 1 shows that for the case of the the specific heat the agreement between the data of the simulation and the theoretical calculation is even better. Note that the shape of $c(T)$ resembles the one expected for a Schottky anomaly and a closer look at Eq. (A.4) shows that the full theoretical expression is indeed very similar to such a functional form (which is $c(T) \propto \beta^{2} \exp (-p J / T)$ ). It is found that the dominant contribution to $c(T)$ does come from clusters in which exactly two spins are coupled ferromagnetically and hence for which the energy needed to flip one spin is $p J$.

Note that using the theoretical approach presented in the Appendix it is also possible to calculate the ferromagnetic susceptibility shown in Fig. 2b. We have found, however, that the theoretical predictions are only in qualitative agreement with the results from the simulations and that significant quantitative differences exist. The reason for this quantitative discrepancy seems to be that this type of approach is not reliable in the presence of anti-ferromagnetic couplings, as a similar calculation for a pure anti-ferromagnetic model (with no disorder) has shown.

Note that from Fig. 3 and 4 we can also conclude that within the accuracy of the data the energy as well as the specific heat are independent of the system size, in contrast to our findings for the mean-field version of the same model [18, 19]. Hence we can conclude tentatively that the system does not show a phase transition at a finite temperature.

The same conclusion emerges from the moments of the spin glass order parameter distribution (Figs. 5 and 6). In particular we see that the first moment shows a trivial $N^{-1 / 2}$ dependence and that $\chi_{\mathrm{SG}}$ shows no sign of a divergence in the whole temperature regime investigated. At the lowest temperatures $\chi_{\mathrm{SG}}$ seems to become constant, which is evidence that the relevant $T$-range has been probed. The value of the constant for $T \rightarrow 0$ is around 3.0. Since for a short range system $\chi_{\mathrm{SG}}(T)$ can be written as a sum of the spin glass pair correlation function over all distances, this result again implies that these correlations are small and very 
short ranged. For high temperatures the $T$-dependence of $\chi_{\mathrm{SG}}$ is compatible with a 1/T-dependence (see inset of Fig. 6), a functional form that is expected for a system below its critical dimension [49]. Also remarkable is the complete absence of finite size effects in this quantity, in strong contrast to the mean field version of the model [18, 19].

Finally we show in Figs. 7 and 8 the temperature dependence of the cumulant $g_{4}(N, T)\{\mathrm{Eq}$. (14) $\}$ and the Guerra parameter $G(N, T)\{\mathrm{Eq}$. (15) $\}$ for different system sizes. One recognizes that with increasing $L$ these quantities converge to zero for all temperatures, which is again consistent with the complete absence of a finite transition temperature for this model.

\section{DYNAMIC PROPERTIES OF THE $\pm J$ POTTS GLASS}

Having discussed the static properties of the $\pm J$ Potts glass, we now investigate its dynamical properties.

In Fig. 9 we show the time dependence of the (average) spin-autocorrelation function $C(t)$, see Eq. (16), for all temperatures investigated. Although the data shown are for $L=10$, we found that within the accuracy of our data the curves are identical to the ones for larger system sizes. This can be seen in the inset of the figure where we compare for $T=1.6$ the curve for $L=10$ with the one for $L=16$. Note that this absence of finite size effects is in strong contrast to the results found for the mean field version of the present model [18, 19].

From the figure we see that the time-dependence of $C(t)$ is rather unusual in that at low $T$ the function does not show a two step relaxation process as in Ising spin glasses, but instead shows two plateaus: One with a height of approximately 0.6 and another one with height 0.1 . Note that normally the presence of such a plateau is associated with the existence of some sort of temporary cage in which the spins are trapped for a certain time. Thus the fact that we now see two plateaus might be taken as an indication that there are (at least) two different type of cages. However, as we will show below, for the present model the reason for the existence of the plateaus is rather different, despite their similarity with the plateaus seen in the mean field version of the same model close to the dynamical

critical temperature $T_{D}$ [9, 10, 11, 18, 19]. Last not least we remark that the existence of further plateaus cannot be ruled out since within the accuracy of our statistics and within the temperature range accessible in our simulation it is not possible to make any reliable statements on this.

In order to measure the lifetimes of these plateaus, we define relaxation times $\tau_{i}$ via the conditions

$$
C\left(t=\tau_{1}\right)=0.4, \quad C\left(t=\tau_{2}\right)=0.08, \quad \text { and } \quad C\left(t=\tau_{3}\right)=0.05 .
$$

Thus, the relaxation times $\tau_{i}$ are obtained from the intersections of the relaxation functions $C(t)$ at the various temperatures with the horizontal dashed straight lines shown in Fig. 9. The time $\tau_{1}$ measures the lifetime of the first plateau, whereas $\tau_{2}$ 
and $\tau_{3}$ measure the one of the second plateau. (The reason for using two different definitions for the latter will be discussed below.)

Many glass forming systems have the property that their time correlation functions obey the so-called time-temperature superposition principle (TTSP) [27. This means that if one plots a time correlation function versus $t / \tau$, where $\tau$ is the $\alpha$-relaxation time, one finds that the correlators for different temperatures collapse onto a master curve. The existence of the TTSP is one of the main predictions of the mode-coupling theory of the glass transition and it follows directly from the solution of the mode-coupling equations [27]. Since the latter are identical to the one derived for the time-dependence for $C(t)$ for the mean field version of the present model, it is of course of interest to check to what extend the TTSP holds for the present short range model as well. That this is indeed the case is demonstrated in Fig. 10 where we plot $C(t)$ vs. $t / \tau_{1}$ and vs. $t / \tau_{2}$. We see that with this type of plot the curves for the different temperatures do indeed fall on top of each other, as expected from the TTSP. (We mention that a plot of $C(t)$ vs. $t / \tau_{3}$ also leads to a superposition of the curves.)

In Fig. 11 we show an Arrhenius plot of the temperature dependence of the relaxation times $\tau_{i}$. From this plot we see that the $T$-dependence of the times $\tau_{2}$ and $\tau_{3}$ is the same and hence we conclude that the exact definition how we defined these relaxation times is irrelevant.

Also included in the graph are straight lines which represent fits to the data. Since these fits give a very good description of $\tau_{i}$ we conclude that the $T$-dependence of $\tau_{i}$ is just an Arrhenius law:

$$
\tau_{i} \propto \exp \left(E_{A}^{(i)} / T\right)
$$

The activation energies are $E_{A}^{(1)} \approx 14.6$ and $E_{A}^{(2)}=E_{A}^{(3)} \approx 28.2$. The interpretation of this observation is in fact quite simple: From Eqs. (11) and (6) we conclude that the breaking of one ferromagnetic bond costs an energy $p \sqrt{2} \approx 14.14 \approx E_{A}^{(1)}$, while overturning a Potts spin which is coupled by two ferromagnetic bonds to neighboring spins would require an energy $2 p \sqrt{2} \approx 28.3$ and this is $E_{A}^{(2)}=E_{A}^{(3)}$.

In order to understand this behavior in more detail, it is illuminating to analyze the relaxation dynamics of single spins. Note that for such an analysis it is necessary to simulate the system for a time that is on the order of $10^{3}$ larger than the longest relaxation time of the system, since only then it is possible to obtain a statistics that is sufficiently accurate. Due to this heavy numerical task, we will present the following analysis only for one realization of the disorder. However, the results for two different samples gave qualitatively the same results.

In Fig. 12 we show the time dependence of $C_{i}(t)$ for three different temperatures $\left(T=2.4,2.0\right.$ and 1.6). Since $L=10$, each plot has $10^{3}$ different curves. One sees immediately that the relaxation dynamics of the system is very heterogeneous in that the typical decay time of the different curves spans many decades and that this range increases rapidly with decreasing $T$. We find that about $40 \%$ of the curves relax to (basically) zero very quickly, i.e. within just a few MCS. This fast 
relaxation is the reason for the presence of the first plateau seen in $C(t)$ at a height of approximately $0.6(=1-0.4)$. For $T=2.4$ (Fig. 12a) most other curves decay to zero within a time of $10^{3} \mathrm{MCS}$. However, if the temperature is decreased to $T=1.6$, see Fig. 12c, we see that the typical relaxation time of these curves has increased to about $10^{4} \mathrm{MCS}$. Thus we conclude that the second step in $C(t)$ is due to the relaxation of these spins. In addition we also recognize from the figure that some spins relax only on the time scale of $10^{6}-10^{7}$ MCS. Thus the existence of the second plateau in $C(t)$ is related to the presence of these slow spins. We also note that the presence of dynamical heterogeneities has also already been observed in a short range Ising spin glass [50, 51]. However, in that case no simple interpretation of the results has been given so far.

Apart from the curves $C_{i}(t)$ that, at low $T$, can be grouped together in a simple way, there are also curves which show a rather complicated time dependence in that they may show a fast decay at short times, one or two plateau(s) at intermediate times, before they decay to zero at long times. Since the total fraction of such curves is only a few (3-4) percent, and the number of such curves that relax only on the longest time scales does not seem to increase with decreasing $T$, we feel that they are not important for the average relaxation dynamics of the system. Therefore we will neglect them in the following.

We have found that the shape of the remaining curves can be described very well by a simple exponential. In addition the $T$-dependence of relaxation time $\tau_{i}^{\prime}$ of each curve is close to an Arrhenius law and therefore we can make the following Ansatz:

$$
\tau_{i}^{\prime}=a_{i} \exp \left(b_{i} / T\right), \quad i \in\{1, \cdots, N\} \quad .
$$

A histogram with the distribution of the parameters $a_{i}$ and $b_{i}$ is shown in Fig. 13 . Note that since we have determined the $\tau_{i}^{\prime}$ at three different temperatures we can use the combinations $(T=1.6$ and $T=2.0)$ as well as $(T=2.0$ and $T=2.4)$ to determine the two parameters $a_{i}$ and $b_{i}$. However, as can be seen from Fig. 13, the two combinations of temperatures give rise to very similar histograms which is evidence that the Ansatz (21) holds and that the way the parameters $a_{i}$ and $b_{i}$ were determined is irrelevant.

While the distribution of $a_{i}$ seems to be continuous without any special features (see inset), the one for $b_{i}$ shows clearly three peaks: One at $b_{i} \approx 0$, one at at $p J$ and a third one at $2 p J$ respectively. Thus we see that the three different types of groups of spins identified in Fig. 12 can also be found in this analysis.

It turns out that all these features of the relaxation behavior can even be understood quantitatively. First we mention our observation that all of the spins that relax quickly do not have ferromagnetic bonds. Recalling that each spin has $z=6$ neighbors and that there are $p=10$ states at disposal, the probability that a spin has only antiferromagnetically coupled neighbors is given by $(1-x)^{6} \approx 0.39$, which accounts for the numerically estimated fraction of $40 \%$ of fast relaxing spins. More 
general, the probability to have exactly $k$ ferromagnetic bonds out of $n$ bonds is

$$
P(k, n)=\frac{n !}{k !(n-k) !} x^{k}(1-x)^{n-k} .
$$

So, a first estimate would be to say that a spin with exactly $k$ ferromagnetic bonds relaxes with a barrier given by $k p J$. Noting that $P(1,6) \approx 0.40, P(2,6) \approx 0.17$, $P(3,6) \approx 0.04$ etc., we note that this approach would predict a second plateau at a height of 0.21 (resulting from the sum of probabilities with $k \geq 2$ ), and also predict a third plateau with a rather large height of about 0.046. Since we do not observe such a third plateau it is necessary to refine the approach: While spins with a single ferromagnetic bond need to overcome a barrier of $p J$, the spins with two ferromagnetic bonds do not necessarily need to overcome a barrier of $2 p J$. E.g., if one of the two spins that are connected ferromagnetically has itself only one ferromagnetic bond, it will relax with a barrier $p J$, and thus can subsequently permit to the first spin with two ferromagnetic bonds also to relax, overcoming a barrier of $p J$ only. The probability to have such an arrangement of bonds is given by $P(2,6)$, that is the probability for the spin to have exactly 2 ferromagnetic bonds with its neighbors, times the probability that one of these two spins has no further ferromagnetic bond with its remaining 5 neighbors, and which is thus $P(0,5)$. This yields $P(2,6) \cdot P(0,5) \approx 0.077$, so that the total percentage of spins relaxing with barriers higher than $p J$ is lowered to 0.13 , very close to the value found for the second plateau from Fig. 9. Extending this reasoning also to spins relaxing with a barrier $3 p J$, one estimates that the height of a third plateau would be around 0.006 , and this is clearly too small to be observable numerically within the accuracy of our data. Note also that these numerical estimates completely ignore the small effects due to the frustrated bonds, which still must be present, as the estimate for the ground state energy shows. Nevertheless, these arguments show that the relaxation of the model is to a large extend accounted for by the behavior of small isolated clusters of correlated spins, and no collective relaxation associated with either dynamic or static transition is needed.

\section{THE GAUSSIAN POTTS GLASS}

We now turn our attention to the case where the couplings between neighboring spins are not given by a bimodal distribution, but by a Gaussian one (see Eq. (7)) . As we will see, this change in the distribution has important implications for the static and dynamic properties of the system. All results discussed in this section were obtained for $L=10$.

In Fig. 14 we show the $T$-dependence of the energy per spin. In agreement with our finding for the $\pm J$ model, this function does not show any sign for the presence of a singularity. At low temperatures we see that (with decreasing $T$ ) the

slope of the curve decreases and that $e(T)$ approaches smoothly its value in the 
ground state. This value is close to -5.49 , the energy of the system if one assumes that all interctions are satisfied, i.e.

$$
e_{\mathrm{gs}}^{\mathrm{appr}}=3\left(\int_{-\infty}^{0} P\left(J_{i j}\right) J_{i j} d J_{i j}-(p-1) \int_{0}^{\infty} P\left(J_{i j}\right) J_{i j} d J_{i j}\right) \quad .
$$

Here $P\left(J_{i j}\right)$ is the distribution given by Eq. (7). The value of $e_{\mathrm{gs}}^{\mathrm{appr}}$ is marked in the figure by the horizontal arrow.

That $T$-dependence of the specific heat is shown in Fig. 15. Similar to the case of the $\pm J$ model, we find a Schottky-like peak at around $T \approx 2$. Since in the Gaussian model there is no gap in the excitation spectrum, by analogy with Ising spin glasses [1], we expect a low temperature behavior $c(T) \propto T$ and $e(T)-e(0) \propto T^{2}$ rather than the behavior proportional to $\exp (-p J / T)$ encountered in the $\pm J$ model. However, since for $T \leq 1.2$ the relaxation times are already rather large, no attempt has been made to verify these expectations numerically.

Since the ferromagnetic bonds now occur with a broad spectrum of energies rather than a single energy $p J$, the argument put forward in the previous section for the existence of a plateau does no longer apply. In Fig. 16 we show the timedependence of $C(t)$ for all temperatures investigated and from this graph we see that in this $T$-range there is no sign for the presence of a plateau. Although for long times and low $T$ the TTSP seems to hold reasonably well, we have not been able to determine the functional form of the time dependence of $C(t)$.

Of course it might be that the absence of the plateau is related to the fact that we are investigating the system at too high temperatures. In order to check for this possibility we have quenched 10 independent sample (i.e. different realisations of disorder) to $T=0.5$ and have annealed the system for $10^{7}$ MCS before we started a production run of $10^{7} \mathrm{MCS}$. Although this time is certainly insufficient to equilibrate the systems at this temperature, it can be expected that for times significanly $(\mathrm{O}(10))$ shorter than $10^{7}$ MCS the correlation function is close to the one in true equilibrium.

The correlation function as calculated after the quench is included in Fig. 16 as well. We see that even at this low temperature there is no well defined plateau. Instead the time dependence seems to be compatible with a logarithmic decay, at least in the time interval $10 \leq t \leq 10^{5}$. Hence we have evidence that for this model the mean field prediction for the existence of a dynamical transition does not seem to hold.

If we define relaxation times similarly as done previously by requiring that $C(t)$ reaches a particular value,

$$
C\left(t=\tau_{1}\right)=0.1, \quad C\left(t=\tau_{2}\right)=0.05, \quad C\left(t=\tau_{3}\right)=0.02
$$

(horizontal dashed lines in Fig. 16) we find again that the $\tau_{i}$ have an Arrhenius temperature dependence (see Fig. 4 in Ref. [38]), but the activation energy seems to increase smoothly the smaller the constant in Eq. (24) is chosen. (We find the activation energies $20.1,16.4$, and 12.8 for $\tau_{3}, \tau_{2}$ and $\tau_{1}$.) Once more, this 
behavior is again expected from a system where one has small clusters (dimers, trimers, etc.) of ferromagnetically coupled Potts spins, and the breaking of this (continuous spectrum) of ferromagnetic bonds causes the extended tails of the time-correlation functions $C(t)$ as seen in Fig. 16.

\section{CONCLUDING REMARKS}

In this paper we have used Monte Carlo simulations to investigate the static and dynamic properties of the 10-state Potts glass model with nearest neighbor random interactions on the simple cubic lattice. We have found that in the temperature range investigated there is no sign for the presence of the dynamical transition from ergodic to nonergodic behavior nor for the static discontinuous glass transition that one finds in the mean field version of this model. Instead the dynamic transition is replaced by a very gradual onset of slow dynamics, described by relaxation times that are compatible with simple Arrhenius laws.

For the case of the $\pm J$ model, the spectrum of relaxation times is essentially discrete - namely times $\tau_{k} \propto \exp (k p J / T)$ with $k=1,2$. These times are related to processes in which a spin is connected to its nearest neighbors via $k$ ferromagnetic bonds that have to be broken for the spin to flip. In contrast to this, the Gaussian model has a spectrum of relaxation times that is continuous, which reflects the fact that also the values of the ferromagnetic bonds are distributed continuously.

A further important difference between the $\pm J$ model and the Gaussian model is that the average spin auto-correlation function of the latter shows, after a fast drop at short time, a smooth decay to zero. In contrast to this, $C(t)$ for the $\pm J$ model shows several steps, the existence of which is related to the discrete spectrum of relaxation times. The auto-correlation function of individual spins is for most spins a simple exponential, with a decay time that is given by one of the relaxation times $\tau_{k}$ mentioned above. Thus, although there is a pronounced dynamical heterogeneity in the model, this heterogeneity is localized on a very small length scale (some spins have only antiferromagnetic bonds to their neighbors and can relax fully unhindered, others are in ferromagnetically coupled dimers, trimers, etc., but the concentration of large clusters is vanishingly small). Due to the strong localization of these excitations it is unlikely that a description of dynamical heterogeneity in terms of mesoscopically large regions ("droplets" where volume and surface terms in their free energy compete, etc. [25, 37]) is applicable, at least for the present model. Instead we have shown in the present work that a description in terms of small clusters of ferromagnetically coupled spins in a background of spins to which they are "coupled" antiferromagnetically is almost quantitatively accurate. (Note that this antiferromagnetic coupling has almost no effect on the cluster, due to the large number of states for the spins that still are available when a bond between them is antiferromagnetic.) E.g. this description allows to explain the $T$-dependence of static quantities like energy, or the specific heat very well, and thus we conclude that in the present model 
the spins are correlated only very weakly. The fact that we are able to give an almost quantitative description of the static as well as dynamic properties of the system in the $T$-range considered makes it also very unlikely that a dynamical or static transition occur at even lower temperatures, although strictly speaking such a possibility can of course not be ruled out.

Hence we conclude that the idea that short range Potts glasses may provide a good model for the structural glass transition problem, exhibiting a rounded version of a dynamic transition (with a crossover from a power-law divergence of the relaxation time to an Arrhenius behavior with a very large barrier) and a (very closely avoided) static transition to a glass phase at a lower but nonzero temperature can not be maintained, at least not for the present model. Instead we think that a better model for the structural glass transition would be a Potts glass with interactions that have a somewhat longer range than in the models studied here.

Very recently it has been suggested that such a close analogy between Potts glasses and structural glasses might exist only if the number of available states is very large, e.g. $p=10^{3}$ [37]. However, we do not see any physical basis for such a claim: The choice $\Delta J=1, J_{0}=-1$ in the model, necessary to avoid the tendency for ferromagnetic ordering, leads to a fraction $x \approx 0.146$ of ferromagnetic bonds, and for larger values of $p$ an even smaller $x$ is needed [12]. However, with such a small fraction of $x$ the properties of the model will be qualitatively the same for $p=10$ and $p=10^{3}$. Frustration effects also cannot change this conclusion - the fraction of frustrated plaquettes with three ferromagnetic bonds and one antiferromagnetic bond simply is too small (note that plaquettes with one ferromagnetic bond but three antiferromagnetic bonds are frustrated in an Ising spin glass, where $p=2$, but not for the large $p$ values considered here). While the idea [37] that one should discuss the applicability of the mean field Potts glass transition scenario to real systems in terms of a Ginzburg criterion [52] is certainly interesting, it seems that it is not useful for the extremely short range case of a nearest neighbor Potts glass. This conclusion is of course not that surprising since our experience with Ising ferromagnets has shown that the Ginzburg criterion tells little about the nearest neighbor model either, although it is useful for understanding properties of Ising models with a large but finite range of the forces [53]. A study of the 10-state Potts glass with random interactions with such medium range could be illuminating, but clearly is computationally very demanding and hence was not attempted here. In any case, our study shows that it is rather difficult to draw analogies between spin-glass-type models and models commonly used to study the problem of the structural glass transition (hard spheres, Lennard-Jones, etc.) even if the description of such models within mean-field looks very similar.

Acknowledgements: This work was supported by the Deutsche Forschungsgemeinschaft (DFG) under grant No. SFB262/D1. We thank the John von Neumann Institut for Computing (NIC) in Jülich for a generous grant of computing 
time on the CRAY-T3E.

\section{APPENDIX}

In this appendix we briefly describe the analytical calculations we used to obtain the (approximate) temperature dependence of the energy $e(T)$ and of the specific heat $c(T)$.

To calculate $e(T)$ we consider a Potts-spin that we will denote by "0". This spin is connected to $2 d$ neighbors ( $d$ is the dimension of the lattice, i.e. in our case $d=3$ ). Assume that out of the $2 d$ bonds that connect the central spin to its $2 d$ neighbors, exactly $k$ are ferromagnetic and that hence $2 d-k$ are anti-ferromagnetic. In the following we will calculate the partition function for such a " $k$-cluster" of $2 d+1$ spins and subsequently its energy. We then make the approximation that the Potts Hamiltonian given by Eq. (四) is a sum of independent clusters (using the distribution $P\left(J_{i j}\right)$ to determine the frequency with which a $k$-cluster occurs).

Using the notation $\Delta:=p J$, we can write the Hamiltonian of a $k$-cluster as

$$
\mathcal{H}_{k}=-\Delta \sum_{i=1}^{k} \delta_{\sigma_{0} \sigma_{i}}+\Delta \sum_{i=k+1}^{2 d} \delta_{\sigma_{0} \sigma_{i}}+2 J(k-d)
$$

The partition function can now easily be obtained as

$$
Z_{k}=p[p-1+\exp (\beta \Delta)]^{k}[p-1+\exp (-\beta \Delta)]^{2 d-k} \exp [\beta 2 J(k-d)],
$$

with $\beta=1 / k_{B} T$. The energy can now be calculated by using the relation $e_{k}(T)=$ $-Z_{k}^{-1} \partial Z / \partial \beta$ and one obtains:

$$
e_{k}(T)=\Delta\left\{\frac{(2 d-k) \exp (-\beta \Delta)}{p-1+\exp (-\beta \Delta)}-\frac{k \exp (\beta \Delta)}{p-1+\exp (\beta \Delta)}\right\}+2 J(k-d)
$$

The specific heat $c_{K}(T)$ can now be calculated using $c_{K}(T)=\partial e_{K}(T) / \partial T$ which gives

$c_{k}(T)=\Delta^{2} \beta^{2}(p-1) \exp (-\beta \Delta)\left\{\frac{(2 d-k)}{[p-1+\exp (-\beta \Delta)]^{2}}-\frac{k}{[(p-1) \exp (-\beta \Delta)+1])^{2}}\right\}$.

If in the real system we pick an arbitrary spin and consider its $2 d$ nearest neighbors there is a probability $W(k)$ that the first spin has exactly $k$ ferromagnet bonds and $W(k)$ is given by:

$$
W(k)=\frac{(2 d) !}{k !(2 d-k) !} x^{k}(1-x)^{2 d-k},
$$


where $x$ is the concentration of ferromagnetic bonds (see Eqs. (2) and (6)). Thus within the approximation considered here, the value of an observable $Y$ of the system (i.e. energy per spin or specific heat) is given by

$$
Y=\sum_{k=0}^{2 d} Y_{k} W(k)
$$

Using this relation we have calculated the temperature dependence of the energy per spin as well as the specific heat that are shown in Figs. 3 and 4 , respectively. 
[1] Binder K and Young A P 1986 Rev. Mod. Phys. 58801

[2] Mézard M, Parisi G and Virasoro M A 1987 Spin Glass Theory and Beyond (Singapore, World Scientific)

[3] Fischer K H and Hertz J A 1991 Spin Glasses (Cambridge, Cambridge University Press)

[4] Young A P (ed) 1998 Spin Glasses and Random Fields (Singapore, World Scientific)

[5] Gardner E 1985 Nucl. Phys. 257 B 747

[6] Kirkpatrick T R and Thirumalai D 1987 Phys. Rev. Lett. 582091

[7] Elderfield D and Sherrington D 1983 J. Phys. C 16 L497, L971, L1169

[8] Gross D J, Kanter I, and Sompolinsky H 1985 Phys. Rev. Lett. 55304

[9] Kirkpatrick T R and Wolynes P G 1987 Phys. Rev. B 368552

[10] Kirkpatrick T R and Thirumalai D 1988 Phys. Rev. B 375342

[11] Thirumalai D and Kirkpatrick T R 1988 Phys. Rev. B 384881

[12] Cwilich G and Kirkpatrick T R 1989 J. Phys. A: Math. Gen. 224971

[13] Cwilich G 1990 J. Phys. A: Math. Gen. 235029

[14] De Santis E, Parisi G, and Ritort F 1995 J. Phys. A: Math. Gen. 283025

[15] Kirkpatrick T R and Thirumalai D 1995 Transp. Theory Stat. Phys. 24927

[16] Peters B O, Dünweg B, Binder K, d'Onorio de Meo M, and Vollmayr K 1996 J. Phys. A: Math. Gen. 293503

[17] Dillmann O, Janke W and Binder K 1998, J. Stat. Phys. 9257

[18] Brangian C, Kob W and Binder K 2001 Europhys. Lett. 53756

[19] Brangian C, Kob W and Binder K 2002 J. Phys. A: Math. Gen. 35191

[20] Parisi G 1997 Complex Behavior of Glassy Systems ed Rubi M and Perez-Vicente C (Berlin, Springer) p. 111

[21] Franz S and Parisi G 1998 Physica A 261317

[22] Mézard M and Parisi G 1999 Phys. Rev. Lett. 82317

[23] Mézard M 1999 Physica A 265359

[24] Parisi G 2000 Physica A 280115

[25] Xia X and Wolynes P G 2001 Phys. Rev. Lett. 865526

[26] Bengtzelius U, Götze W and Sjölander A 1984 J. Phys. C 175915

[27] Götze W 1990 Liquids, freezing and the glass transition ed Hansen J P, Levesque D and Zinn-Justin J (Amsterdam: North Holland) p. 287

[28] Götze W and Sjögren 1992 Rep. Prog. Phys. 55241

[29] Götze W 1999 J. Phys.: Condens. Matter 11 A1

[30] Kob W 1999 J. Phys.: Condens. Matter 11 R 85

[31] Kauzmann W 1948 Chem. Rev. 43219

[32] Gibbs J H and Di Marzio E A 1958 J. Chem. Phys. 28373

[33] Adam G and Gibbs J H 1965 J. Chem. Phys. 43139

[34] Gutzow I and Schmelzer J 1995 The Vitreous State (Berlin: Springer)

[35] Stillinger F H 1988 J. Chem. Phys. 887818 
[36] Wolfgardt M, Baschnagel J, Paul W and Binder K 1996 Phys. Rev. E 541535

[37] Eastwood M P and Wolynes P G 2002 preprint cond-mat/0206066

[38] Some of our results have been briefly presented in a preliminary report in Brangian C, Kob W and Binder K 2002 Europhys. Lett. 59, 546

[39] Landau D P and Binder K 2000 A Guide to Monte Carlo Simulations in Statistical Physics (Cambridge, Cambridge University Press)

[40] Binder K 1997 Rep. Progr. Phys. 60487

[41] Wu F Y 1982 Rev. Mod. Phys. 54235

[42] Zia R K P and Wallace D J 1975 J. Phys. A: Math. Gen. 81495

[43] Scheucher M, Reger J D, Binder K and Young A P 1990 Phys. Rev. B 426881

[44] Guerra F 1996 Int. J. Mod. Phys. B 101675

[45] Marinari E, Naitza C, Zuliani F, Parisi G, and Picco M 1998 Phys. Rev. Lett 81 1698

[46] Sillescu H 1999 J. Non-Cryst. Solids 24381

[47] Ediger M D 2000 Annu. Rev. Phys. Chem. 5199

[48] Richert R 2002 J. Phys.: Condens. Matter 14 R703

[49] Binder K and Reger J D 1992 Adv. Phys. 41547

[50] Glotzer S C, Jan N, Lookman T, MacIsaac A B, and Poole P H 1998 Phys. Rev. E 577350

[51] Ricci-Tersenghi F and Zecchina R 2000 Phys. Rev. E 62 R7567

[52] Ginzburg V 1960 Sov. Phys. Sol. State 21824

[53] Binder K and Luijten E 2001 Phys. Rep. 344179 


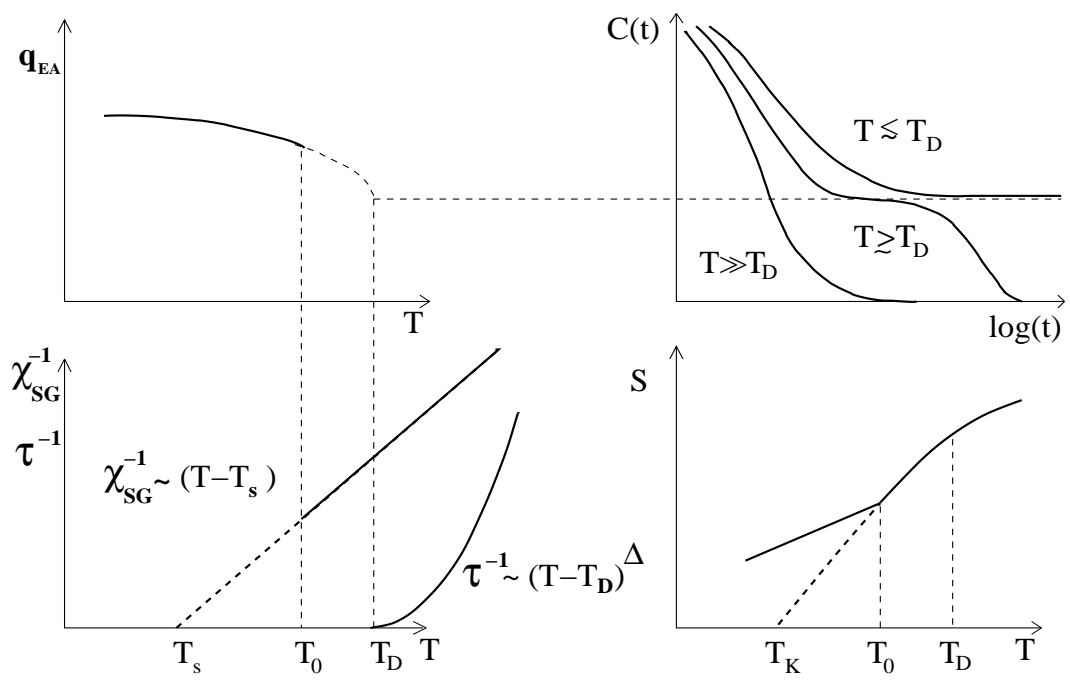

FIG. 1: Schematic sketch of the thermodynamic and dynamic transitions in a meanfield $p$-state Potts glass model with $p>4$ states. The spin glass order parameter $q_{E A}$ is non-zero only for $T<T_{0}$ and jumps to zero discontinuously at $T=T_{0}$. The spin glass susceptibility $\chi_{\mathrm{SG}}$ follows a Curie-Weiss type relation with an apparent divergence at a "spinodal temperature" at $T_{s}<T_{0}$, and hence is still finite at $T_{0}$. The relaxation time $\tau$ diverges according to a power-law $\tau \propto\left(T-T_{D}\right)^{-\Delta}$ at a temperature $T_{D}>T_{0}$. This divergence is due to the occurrence of a long-lived plateau of height $q_{E A}$ in the time-dependent spin auto-correlation function $C(t)$. The entropy $s(T)$ per spin has no singularity at $T_{D}$, but shows a kink singularity at $T_{0}$ (thus there occurs no latent heat, which would mean an entropy jump). The extrapolation of the high temperature branch of the entropy would vanish at a temperature $T_{K} \leq T_{0}$. 

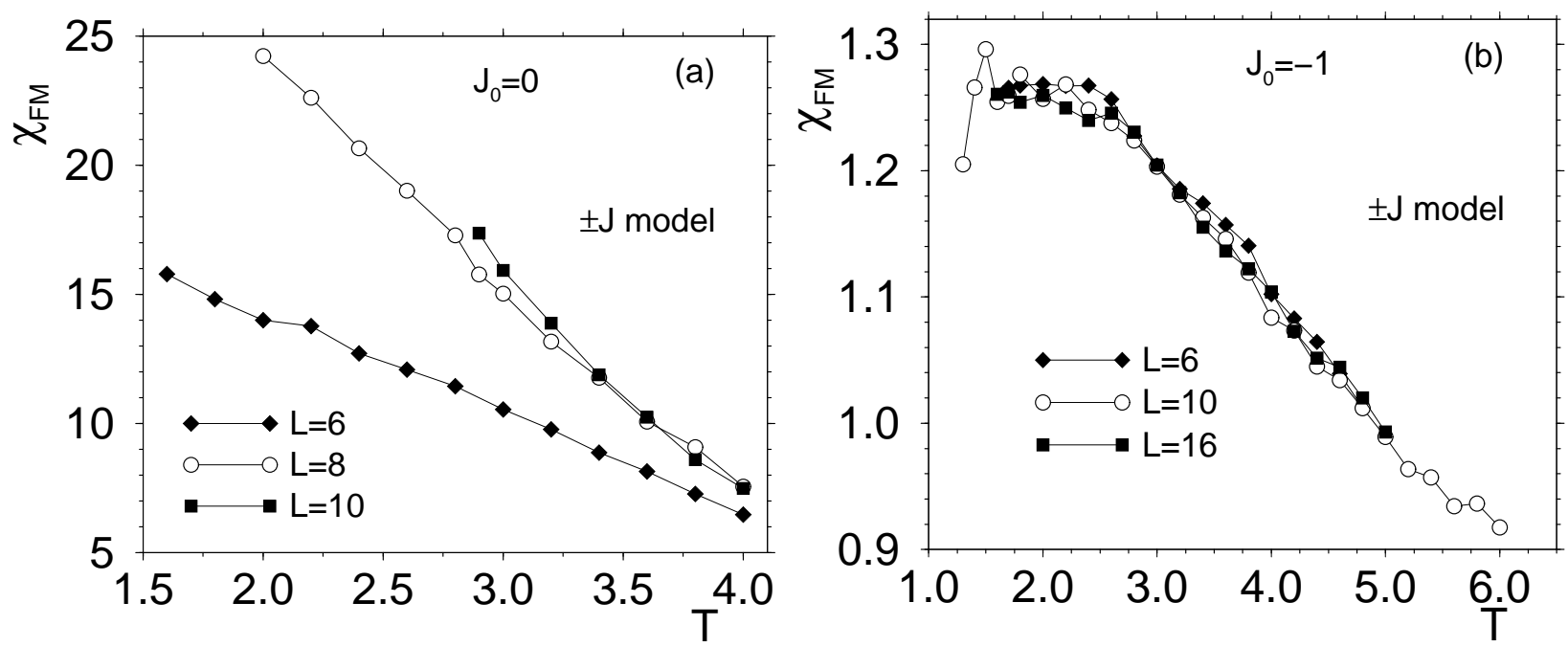

FIG. 2: Temperature dependence of the ferromagnetic susceptibility $\chi_{F M}=N\left[\left\langle m^{2}\right\rangle\right]_{\mathrm{av}}$ in the $p=10$ Potts glass for (a) $J_{0}=0$ and (b) $J_{0}=-1$. The three curves correspond to different system sizes.

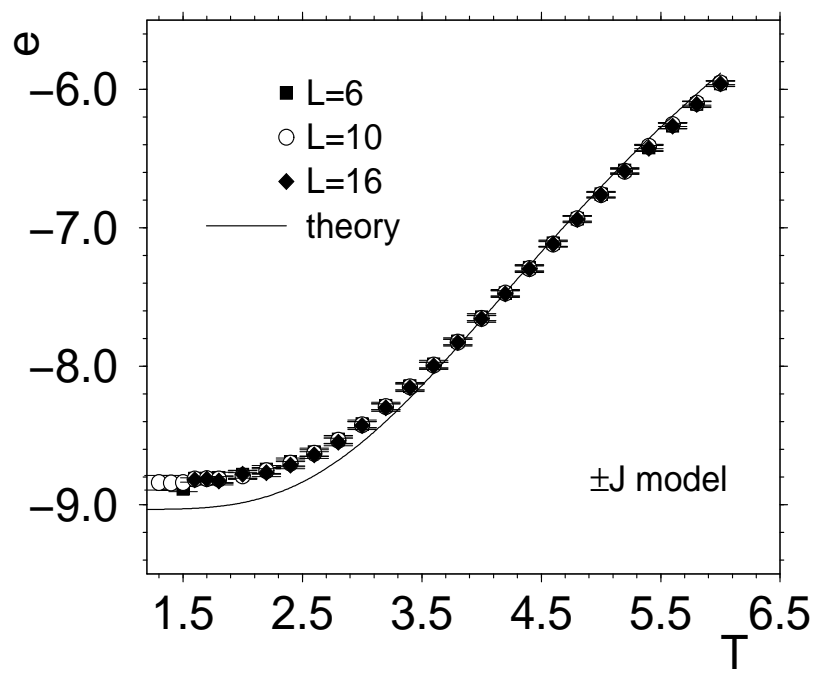

FIG. 3: Temperature dependence of the energy per spin $e(T)$ for three lattice sizes as indicated in the figure. Note that for $T \geq 1.5$ the average $[\cdots]_{\text {av }}$ was realized by averaging over 100 independent realizations for $L=6$ and $L=10$, and by averaging over 50 independent realizations for $L=16$. For $T=1.3$ and $T=1.4$, only $L=10$ was studied, and only 10 realizations were used, since 10 times longer runs than usual were necessary. The solid curve is the theoretical prediction using an approach of independent clusters (see main text for details). 


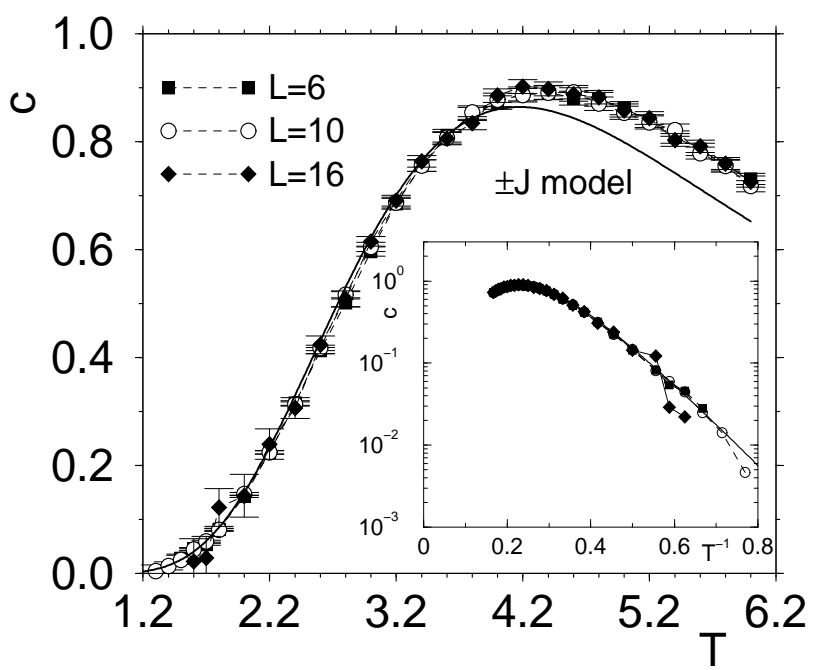

FIG. 4: Specific heat per spin $c(T)$ of the bimodal Potts glass plotted vs. temperature for three lattice sizes, as indicated in the figure. The bold curve is the result of a theoretical calculation using an approach of independent clusters (see main text for details). The inset shows a lin-log plot of c(T) vs. $1 / T$, in order to show the $T$-dependence at low temperatures.

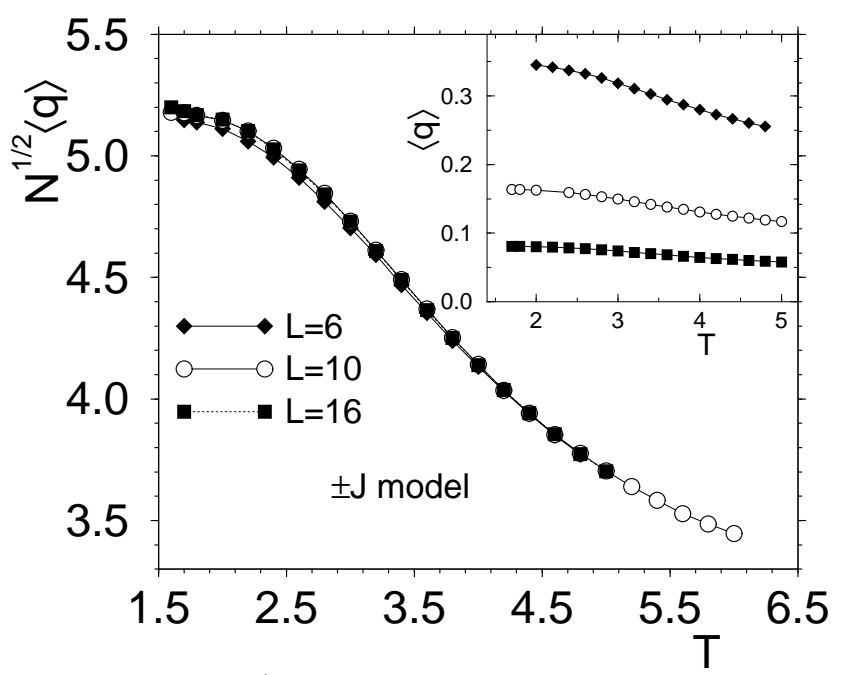

FIG. 5: Scaled first moment $N^{1 / 2}\langle q\rangle$ of the order parameter plotted as a function of temperature. Different curves correspond to the different choices of $L$, as indicated. The inset shows the $T$-dependence of $\langle q\rangle$. 


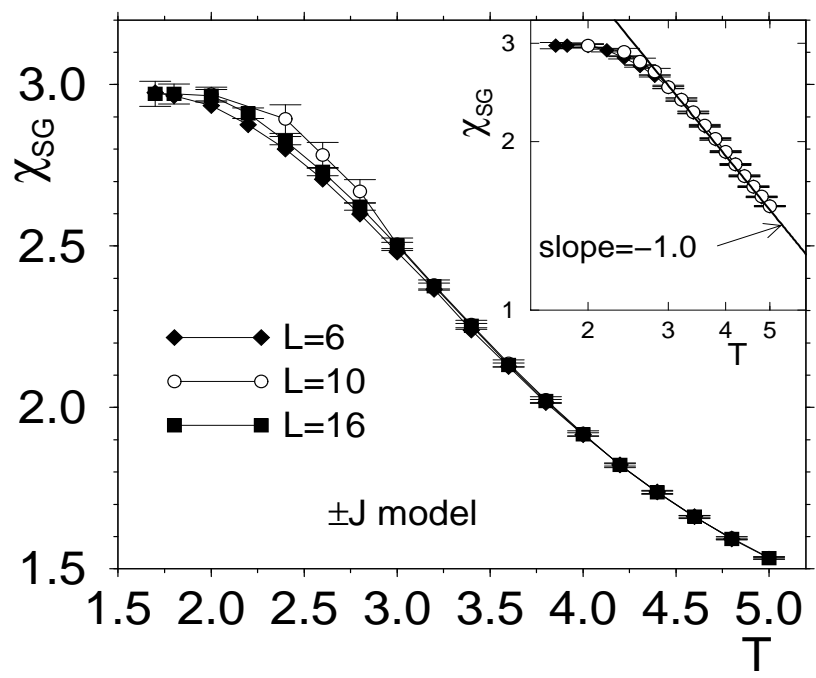

FIG. 6: Spin glass susceptibility $\chi_{\mathrm{SG}}$ as defined in Eq. (13) as a function of temperature. The inset shows the same data in a log-log plot, to demonstrate that at high $T$ we have $\chi_{\mathrm{SG}} \propto 1 / T$.

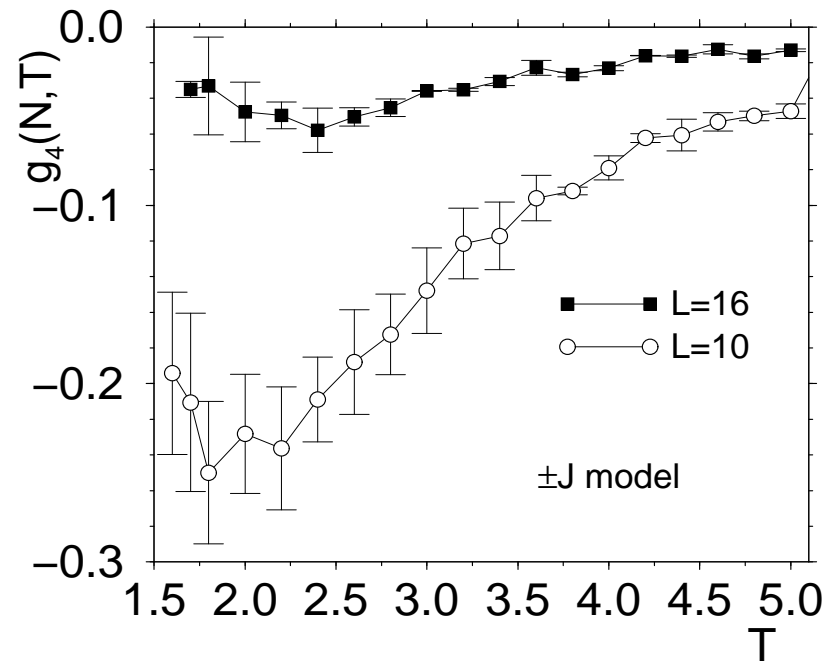

FIG. 7: Plot of the reduced fourth-order cumulant $g_{4}(N, T)$ versus temperature, for $L=10$ (open circles) and $L=16$ (filled squares). The data for $L=6$ were rather noisy and therefore were not included. 


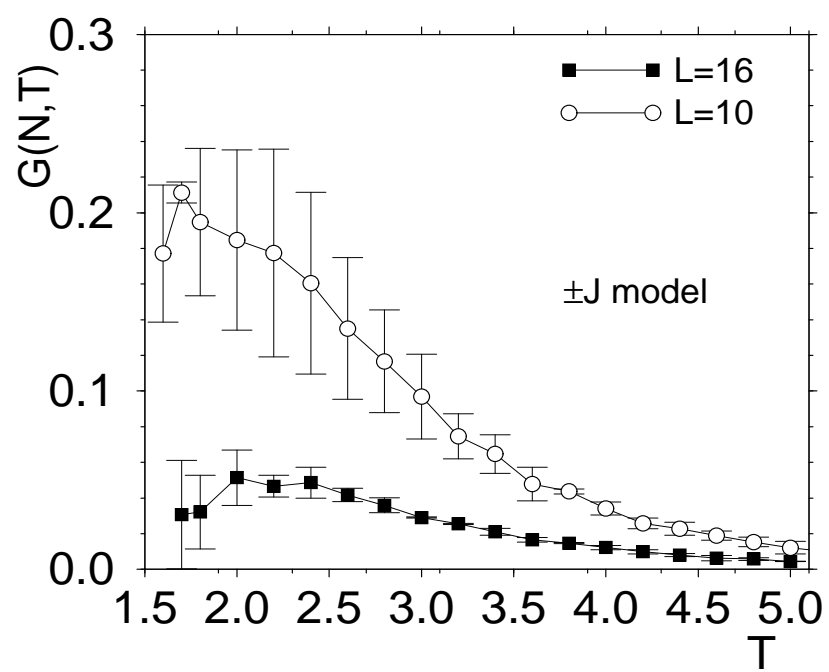

FIG. 8: Plot of the Guerra parameter $G(N, T)$ versus temperature for $L=10$ (open circles) and $L=16$ (filled squares).

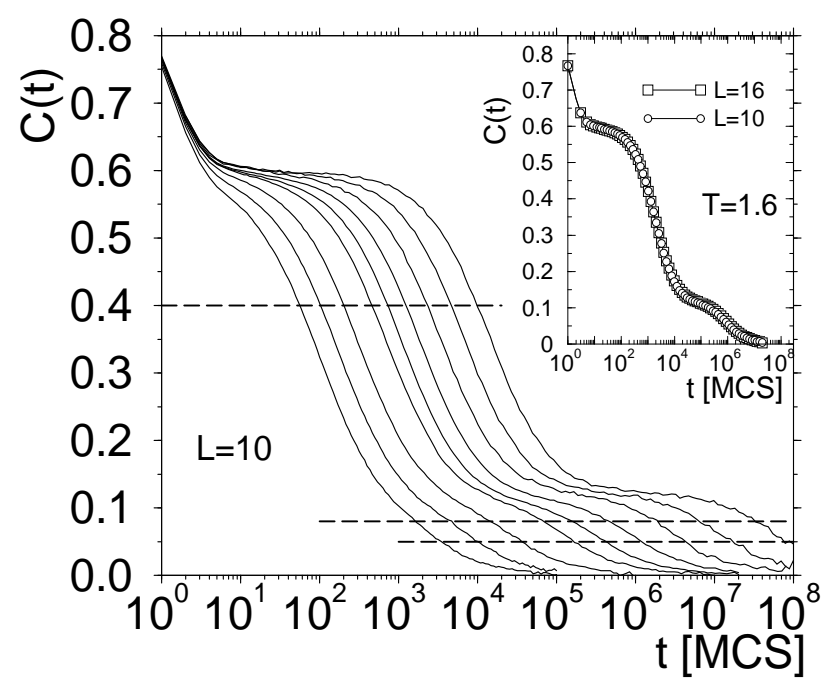

FIG. 9: Time dependence of the spin auto-correlation function of the $\pm J$ Potts glass for $L=10$ and various temperatures (left to right: $T=2.4,2.2,2.0,1.8,1.7,1.6,1.5,1.4$, and 1.3). The three horizontal dashed lines are used to define the relaxation times $\tau_{i}$ (see main text for details). In the inset we compare $C(t)$ for $L=10$ and $L=16$ at $T=1.6$. 

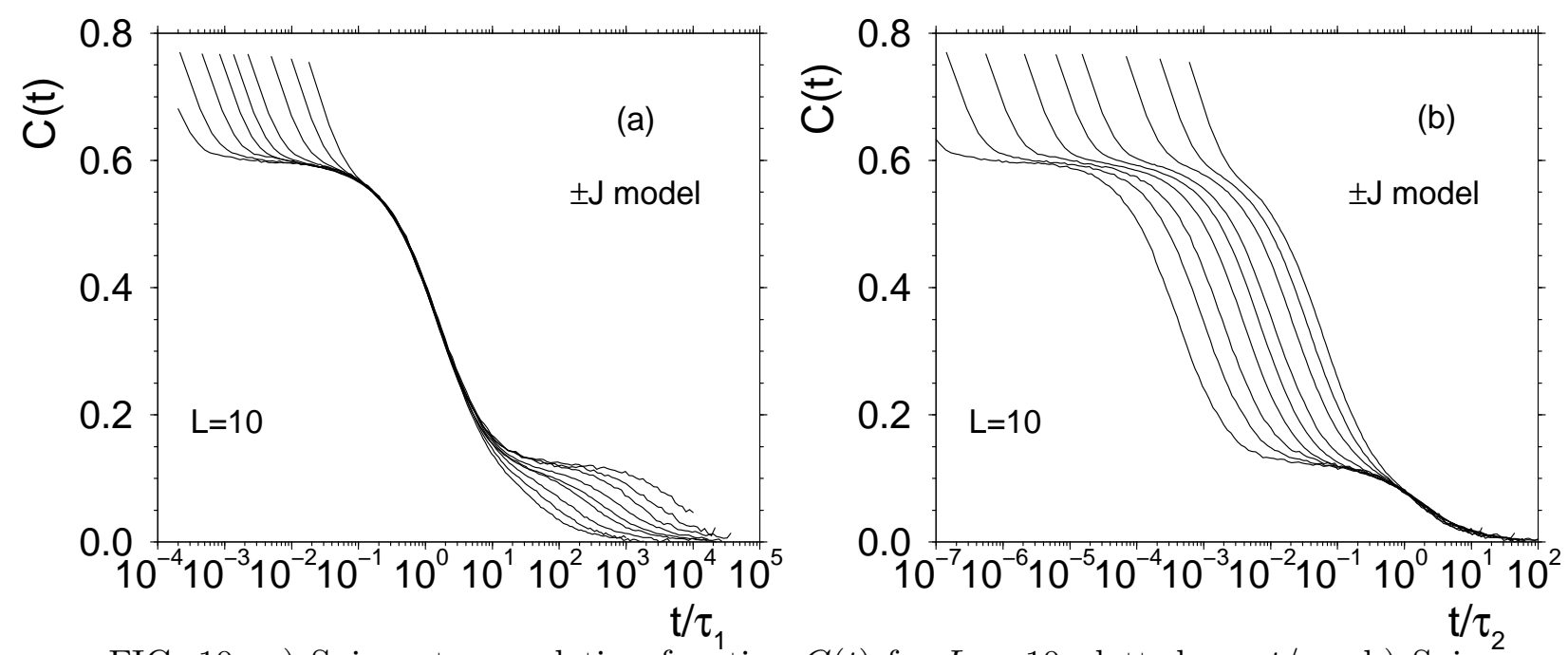

FIG. 10: a) Spin auto-correlation function $C(t)$ for $L=10$ plotted vs. $t / \tau_{1}$. b) Spin auto-correlation function $C(t)$ for $L=10$ plotted vs. $t / \tau_{2}$. The data for $C(t)$ of this figure are identical to the ones shown in Fig. 9 .

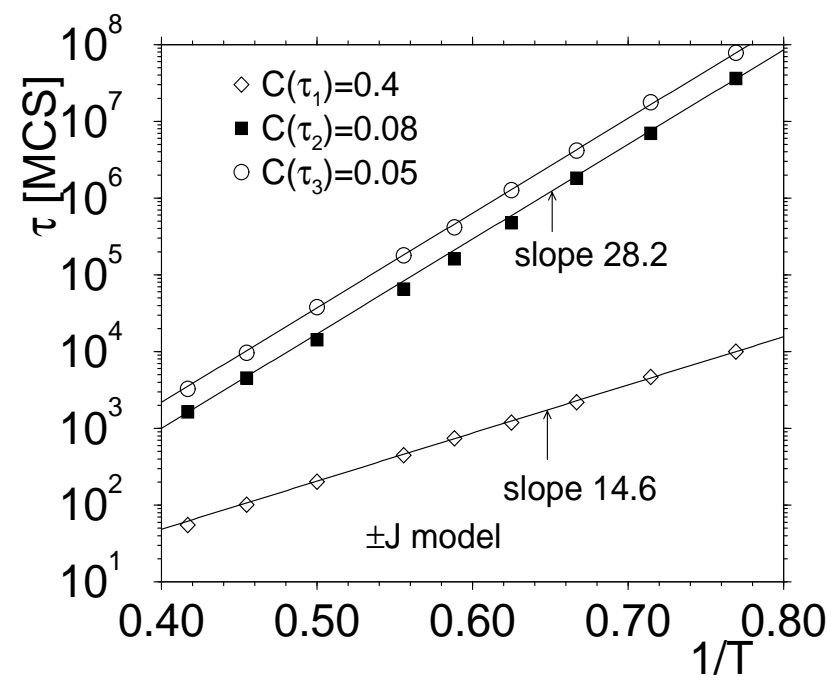

FIG. 11: Arrhenius plot of the relaxation times $\tau_{1}, \tau_{2}$, and $\tau_{3}$ defined in Eq. (19), for the $\pm J$ Potts glass. The straight lines are fits with an Arrhenius law and from their slopes the activation energies $E_{A}^{(i)}$, see Eq. (20), are estimated. 

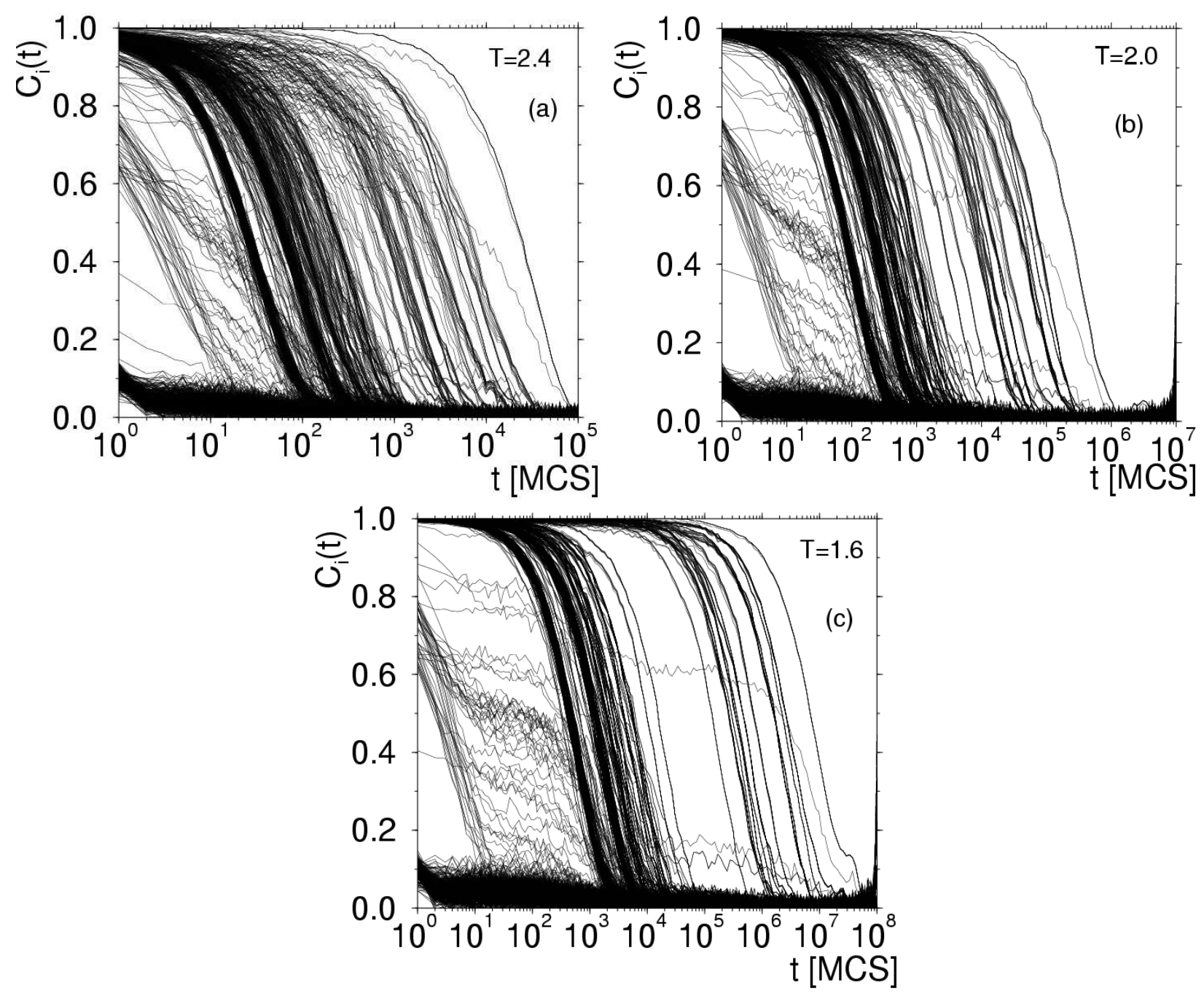

FIG. 12: Time dependence of the relaxation function $C_{i}(t)=(p-1)^{-1}\left\langle\vec{S}_{i}\left(t^{\prime}\right) \cdot \vec{S}_{i}\left(t^{\prime}+t\right)\right\rangle$ of individual spins of the $\pm J$ Potts glass for $L=10$ at three different temperatures: (a) $T=2.4$, (b) $T=2.0$, and (c) $T=1.6$. 


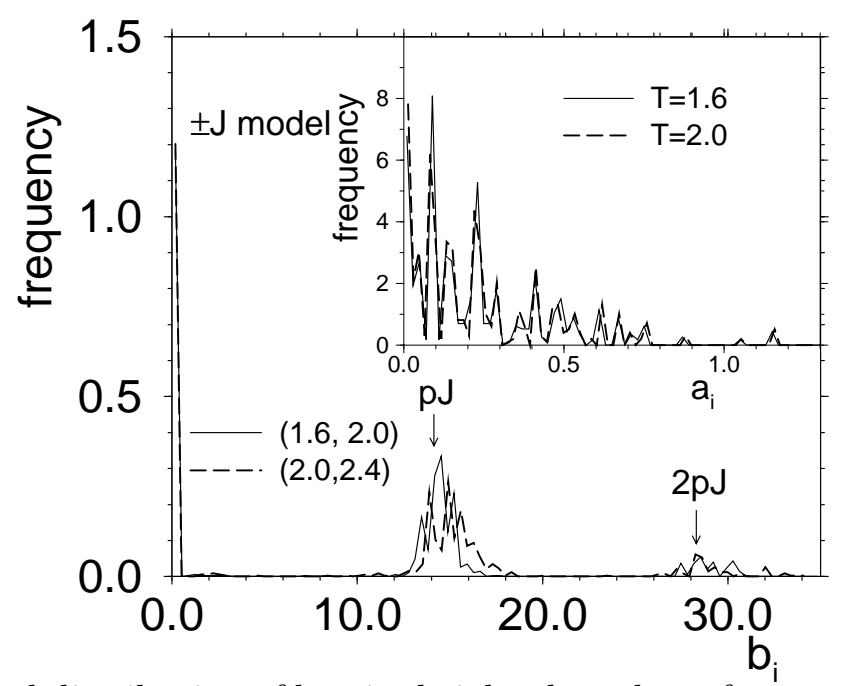

FIG. 13: Normalized distribution of barrier heights $b_{i}$ and pre-factors $a_{i}$ (inset) obtained from fitting the Arrhenius relation, Eq. (21), to the relaxation times $\tau_{i}^{\prime}$ of individual spins, using either the two temperatures $T=1.6,2.0$ (full curves) or $T=2.0,2,4$ (dashed curves).

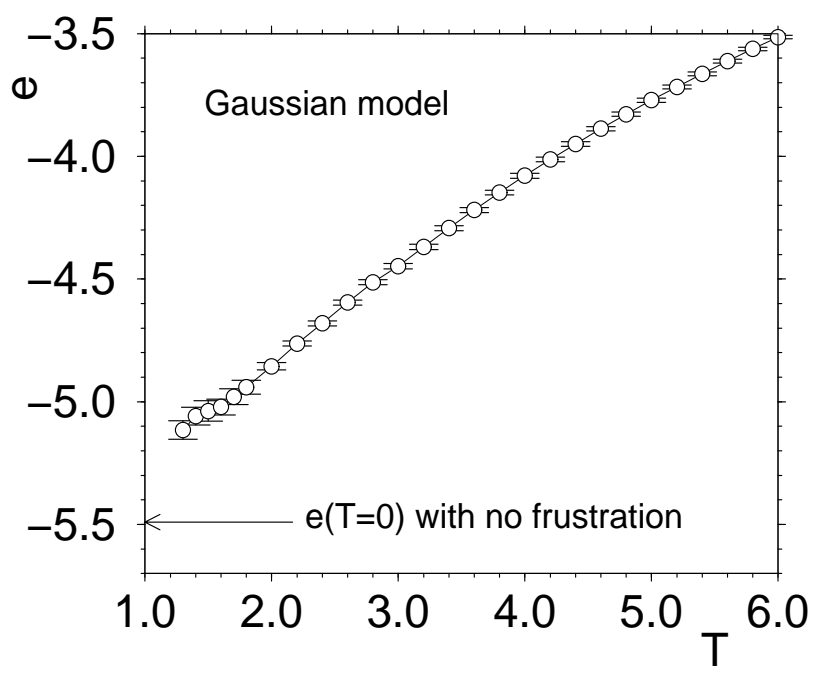

FIG. 14: Energy per spin $e(T)$ of the 10-state Gaussian Potts glass plotted versus temperature for $L=10$. The arrow indicates the estimate of the ground state energy under the assumption that there are no frustration effects (see Eq. (23)). 


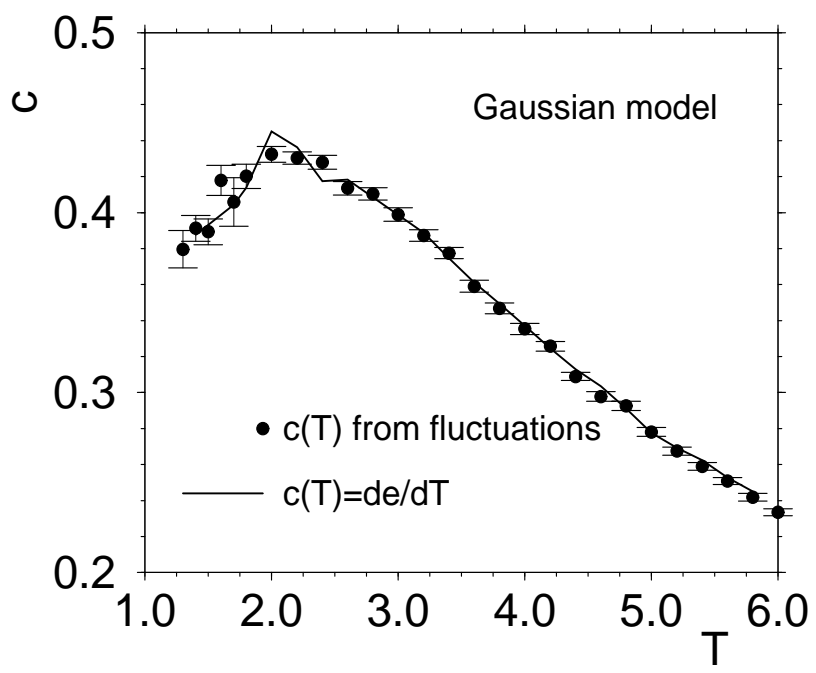

FIG. 15: Specific heat $c(T)$ per spin of the 10-state Gaussian Potts glass plotted versus temperature for $L=10$. Points are from energy fluctuations, while the broken line is a numerical derivative of the curve $e(T)$ shown in Fig. 14.

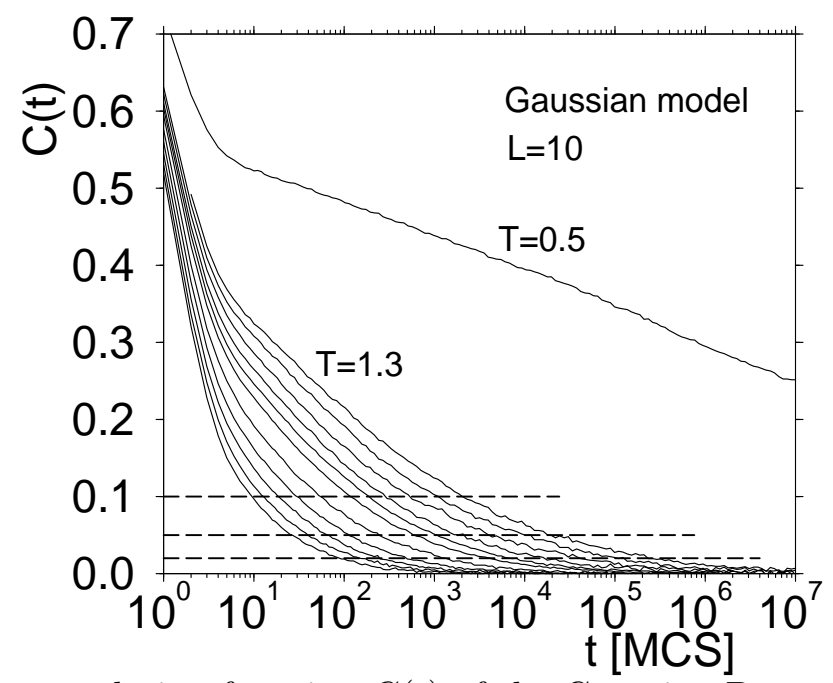

FIG. 16: Spin auto-correlation function $C(t)$ of the Gaussian Potts glass plotted versus time for $L=10$. The temperatures are (from left to right): $T=2.8,2.6,2.4,2.2,2.0$, $1.8,1.7,1.6,1.5,1.4,1.3$ and 0.5 . The horizontal dashed lines are used to define the relaxation times $\tau_{i}$ given in Eq. (24). Note that the data for $T=0.5$ is not in equilibrium (see main text for details). 